\title{
Delayed onset of partial epilepsy of temporal lobe origin following acute clioquinol encephalopathy
}

\author{
T M FERRIER, * A C SCHWIEGER, $†$ M J EADIE $\ddagger$ \\ From the Department of Medicine, Royal Brisbane Hospital, ${ }^{*}$ Brisbane, Department of Neurology, Prince \\ Henry Hospital, $\uparrow$ Melbourne, Department of Medicine, University of Queensland, $\ddagger$ Brisbane, Australio
}

SUMMARY Twelve cases have been reported in the literature to have developed acute, largely reversible, global amnesic syndromes some hours after ingesting substantial doses of clioquinol. Two of these cases presented again after asymptomatic intervals of some 12 years. Both had recently developed typical clinical and electroencephalographic evidence of partial epilepsy originating in a temporal lobe. The similarity of clinical events in these two subjects, the absence of any other known cause for their epilepsy and the fact that, in mice, acute clioquinol overdosage causes hippocampal and amygdaloid injury, suggest that the drug was responsible for both the acute encephalopathy and the epilepsy of delayed onset.

Until comparatively recent times clioquinol (a halogenated hydroxyquinoline derivative) was widely used as an intestinal anti-infective agent. However, following the identification of the drug as the possible cause of the syndrome of subacute neurotoxicity ${ }^{1}$ clioquinol has largely disappeared from use in many countries, except when employed in topical preparations. While the subacute neurotoxicity syndrome in its various facets appears to be dose-related and associated with continued intake of the drug, a syndrome of acute neurotoxicity arising from a short period of high dosage clioquinol intake is also known. This latter disorder resembles the clinical picture of transient global amnesia, though the episodes are more prolonged. To $1984 \mathrm{Kaeser}^{2}$ was able to trace 12 reports of this particular condition in the literature, including two from Australia. Some 13-14 years after these two Australian cases presented with acute encephalopathic syndromes, each was referred again with partial epileptic seizures of recent onset. The present paper describes these events, and considers the relationship between the earlier clioquinol encephalopathy and the subsequent epilepsy.

Address for reprint requests: Professor $M$ Eadie, Clinical Sciences Building, Royal Brisbane Hospital, Brisbane 4029, Australia.

Received 24 April 1986.

Accepted 23 May 1986

\section{Case reports}

Case 1

One evening in 1971 a 43 year old housewife took a single oral dose of 1.5 gram clioquinol after drinking possibly contaminated water while holidaying in Africa. She appeared normal on waking next morning, but half an hour later became restless and confused, could not recognise her husband and could not work out how to eat her breakfast. She experienced headache and vomiting, but was afebrile, without neck stiffness or focal neurological signs. CSF examination was normal. She remained conscious, but asked questions repeatedly and could not remember the answers given to her. On the third day of illness her headache lessened and her mental state began to improve. On the tenth day of illness she remained unusually drowsy: she could recognise her family and friends, though her recent memory remained patchy. She was aware of her memory problem. Her EEG and clinical neurological examinations were normal. Over the next week there was further recovery in memory, though with persisting amnesia for the acute episode.

The patient again presented at the age of 57 years. Over the intervening period her husband, a medical practitioner, considered that her memory had never been as good as it was prior to the acute episode. However, she had led a full and independent family and social life. Recently, once while playing bridge and once while watching tennis, her friends had noted her having brief lapses, lasting about 30 seconds, in which she was immobile and failed to respond to conversation. Some months earlier she had experienced some vague "dizzy" turns, which ceased spontaneously. There 


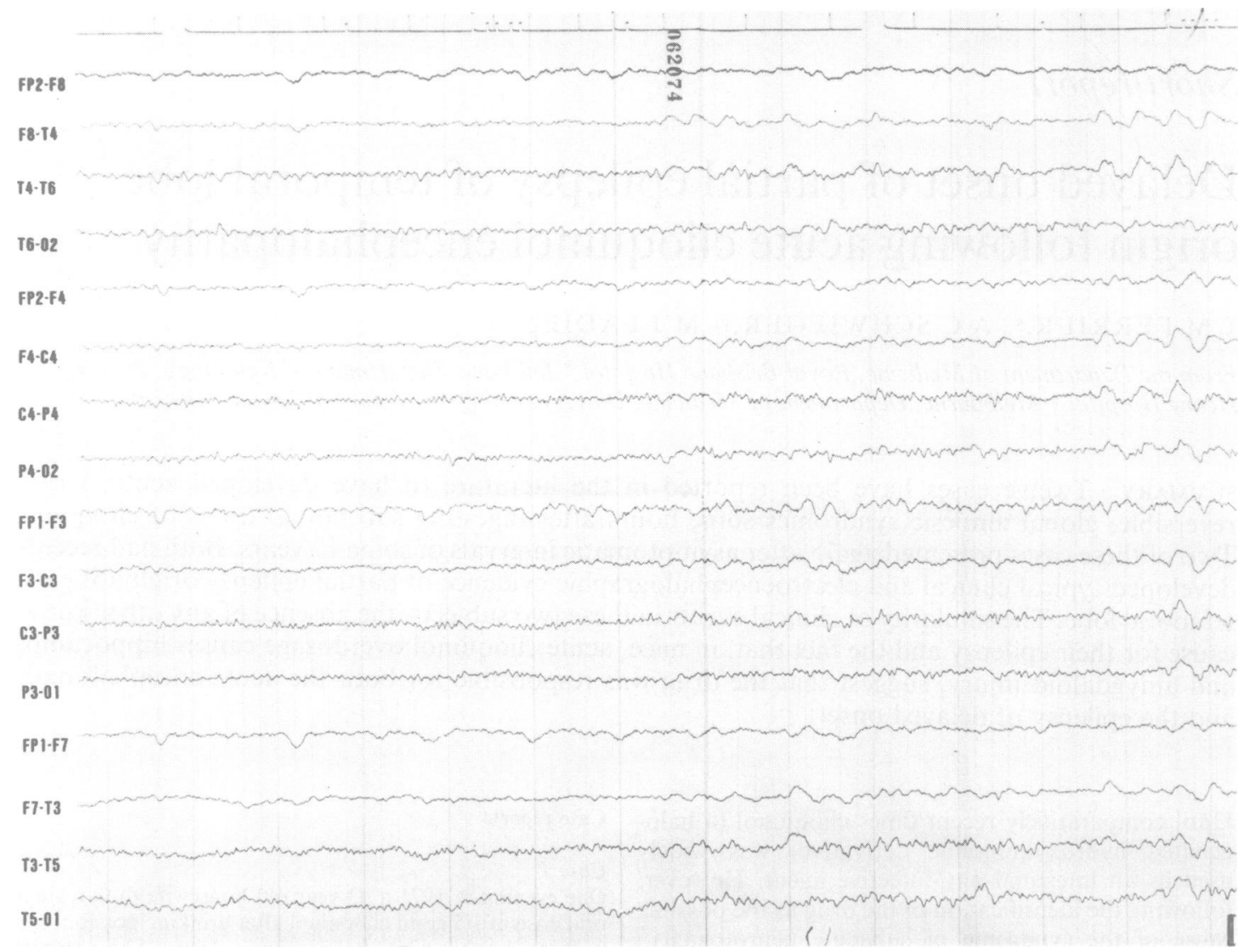

Fig EEG of Case 2 showing isolated sharp waves in the right posterior temporal region occurring in the earlier part of the segment of the recording with bilateral paroxysmal polymorphic sharp and slow wave activity then appearing. (Bar $=50 \mu V)$

were no abnormalities on neurological or general physical examination, except that she made some curious mistakes about personal details. For example, she gave her age as 57 , and her date of birth as 1921 , but when taxed with this discrepancy explained that she had given her husband's date of birth rather than her own.

A few days later the patient's married daughter, a medical practitioner, phoned to say her mother had been having dozens of brief episodes in which she would stand still, unresponsive to conversation, and if the daughter then placed her hand on the mother's arm fine twitching could be felt. Although an EEG at the time of presentation had appeared normal, an EEG several months earlier, at the time of the dizzy turns, had shown occasional paroxysmal discharges in the right anterior temporal region, and her CT head scan had then shown a little symmetrical cortical atrophy in the regions of the frontal poles and islands of Reil, and on the inferior surface of the right temporal lobe.

It was felt there were sufficient grounds for a diagnosis of partial epilepsy probably arising in the right temporal lobe, due to previous damage to this region, possibly occurring at the time of the clioquinol encephalopathy. Therapy with carbamazepine in a dose which yielded a plasma drug concen- tration of $16 \mu \mathrm{M} /$ litre caused cessation of the lapses and a conspicuous improvement in the patient's memory.

Case 2

In 1972 this 32 year old airline pilot took 4 gram of clioquinol orally over a few hours to treat an acute episode of abdominal pain and nausea. Next day he became drowsy, with a slight headache. Though fully alert, he was disorientated in time and place with a severe deficit in recent memory and some defects in more remote memory. Clinical neurological examination was otherwise normal, and there were no abnormalities in his EEG or CSF. His memory difficulties and disorientation were clearing by the third day of his illness, though there were still gaps in his recall two weeks after the onset and it was a month before he could return to work.

His subsequent presentation occurred 13 years later, at the age of 45 years. Over the past year he had experienced a number of episodes which he described as "complete mental shut downs", lasting some seconds, during which he could not remember what occurred, was immobile, looked vacant and was unable to speak. He admitted to a continuing problem with intermittent unreliability of short term memory since the 1972 episode. Clinical examination, EEG and CT 
head scan were normal. Partial epilepsy of temporal lobe origin was suspected, and this diagnosis became very probable when his wife described an episode in which he broke off conversation, made "clearing" noises in his throat, moved his mouth as though "smacking the lips", and then recovered. A second EEG showed paroxysmal activity probably arising in the right mid-temporal region (fig 1).

\section{Discussion}

The two patients reported here, and described previously, ${ }^{2}$ suffered the typical transient global amnesia syndrome which is the main feature of the acute encephalopathy associated with short-term intake of relatively high doses of clioquinol. Subsequent authors $^{3-5}$ have accepted these two cases as unequivocal examples of the syndrome. They showed the characteristic history of intake of a substantial amount of clioquinol over a relatively short period followed, after a lapse of 12 to 24 hours, by the comparatively rapid onset of amnesia lasting for several days in the presence of relatively preserved alertness. As in other reported cases recovery occurred as time passed, though there was enduring amnesia for the period of the acute episode. The pathological basis for the disorder in humans is unknown, as no case has come to necropsy. However, high clioquinol dosage over a short period may cause acute cerebral symptoms in animals of several species. ${ }^{5}$ At necropsy in mice Puschner and Frankhauser ${ }^{6}$ have observed selective regional damage in the amygdaloid nuclei with changes in Ammon's horn, particularly in the large pyramidal cells. If clioquinol caused changes in these same structures in man, they would provide a very adequate explanation for the global amnesia syndrome, since intact hippocampal function appears necessary for short-term storage of new memories.

Clearly such hippocampal and amygdaloid changes would also provide a structural basis for disinhibiting surviving hippocampal neurons so that they might initiate epileptic discharges. Both of the patients here reported developed what could be diagnosed with reasonable certainty on clinical and electroencephalographic grounds as partial epilepsy originating in a temporal lobe. It is tempting to speculate that in these two cases clioquinol intake led to acute bilateral hippocampal damage which caused an incompletely reversible failure of memory and which also set the stage for the subsequent development of epilepsy originating in or near the damaged structures. There is no direct proof of this postulated series of events, but no other causes for the epilepsies are apparent: the lapse of many years between neural injury and the onset of clinical epilepsy is not unusual and, ignoring the possibility of coincidence, the similarities of the latent intervals in the two subjects provide further support for the idea of a pathogenesis common to both persons. It is not known whether epilepsy has developed in other reported cases of acute clioquinol encephalopathy in humans. In animals, convulsions may occur in the acute phase of clioquinol toxicity, and it is unlikely that such animals would remain under observation long enough to see if delayed onset epilepsy developed, if the latent period is really of the order of a decade.

Although clioquinol intoxication cannot be proven the basis of the epilepsy in these two patients, the similarity of events in both subjects makes the earlier brief exposure to the drug a likely cause. When clioquinol is no longer marketed in many countries, the drug is not likely to be responsible for further neurotoxicity or epileptogenesis in humans. However the occurrences in the two patients here reported seem worth recording for their intrinsic interest, and because clioquinol may prove a tool for producing experimental epilepsy in animals.

\section{References}

1 Tsubaki T, Honma Y, Hoshi M. Neurological syndrome associated with clioquinol. Lancet 1971;1:696-7.

2 Ferrier TM, Eadie MJ. Clioquinol encephalopathy. Med J Austr 1973;1:1008-9.

3 Baumgartner G, Gawel MJ, Kaeser HE, et al. Neurotoxicity of halogenated hydroxyquinolines: clinical analysis of cases reported outside Japan. J Neurol Neurosurg Psychiatry 1979;42:1073-83.

4 Kaeser HE. Transient global amnesia due to clioquinol. Acta Neurol Scand 1984;70(Suppl 100):175-9.

5 Mumenthaler M, Kaeser HE, Meyer A, Hess T. Transient global amnesia after clioquinol. Five personal observations from outside Japan. $J$ Neurol Neurosurg Psychiatry 1979;42:1084-90.

6 Puschner H, Frankhauser R. Neuropathologische Befunde bei experimenteller Vioform-Vergiftung der weißen Maus. Schweizer Arch Tierheilkunde 1969; 111:371-9. 